\title{
Identification of non-canonical Wnt receptors required for Wnt- 3a-induced early differentiation of human neural stem cells
}

Nora Bengoa-Vergniory ${ }^{1}{ }^{\wedge}$, Irantzu Gorroño-Etxebarria ${ }^{1}$, Inmaculada LópezSánchez ${ }^{1}$, Michele Marra ${ }^{1}$, Pierluigi Di Chiaro ${ }^{1}$ and Robert Kypta ${ }^{1} 2^{*}$

${ }^{1}$ Cell Biology and Stem Cells Unit, CIC bioGUNE, Bilbao, Spain 2Department of Surgery and Cancer, Imperial College London, UK ${ }^{\wedge}$ Present address: Department of Physiology, Anatomy and Genetics, Oxford University, UK

* Corresponding author email: rkypta@cicbiogune.es, r.kypta@imperial.ac.uk phone: +34944061 321, +442075941928

fax: +34944061301

Keywords: Wnt signaling pathways, neural stem cells 


\section{ABSTRACT}

Wnt proteins preferentially activate either $\beta$-catenin-dependent or $\beta$-cateninindependent signals, but the activity of a particular Wnt also depends on cellular context and receptor availability. We previously reported that Wnt-3a induces neural differentiation of human embryonic stem cell-derived neural stem cells (NSCs) in a $\beta$-catenin-independent manner by activating a signal involving JNK and the AP-1 family member ATF-2. Here, we report the results of a gene silencing approach to identify the Wnt receptors that mediate this response to Wnt-3a. Silencing of ROR2 increased neuronal differentiation, as measured by expression of the genes DCX, NEUROD1 and NGN1, suggesting ROR2 signals normally prevent differentiation. Silencing of the other Wnt receptors singly did not affect Wnt-3ainduced neuronal differentiation. However, pair-wise silencing of ROR1 and FZD4 or FZD5 and of LRP6 and FZD4 or FZD5 inhibited neuronal differentiation, as detected by reductions in the expression of neuronal genes and immunocytochemical detection of DCX and NEUROD1 and DCX. Ectopic expression of these receptors in HEK 293 cells increased ATF2-dependent transcription. In addition, ROR1 co-immunoprecipitated with FZD4 and LRP6 in transfected HEK 293 cells and colocalized with FZD4 and with LRP6 at the cell surface of transfected L cells. Wnt-3a did not appear to affect these interactions but did alter the interactions between LRP6 and FZD4/5. Together, these observations highlight roles for ROR1, LRP6, FZD4 and FZD5 in neural stem cell differentiation and provide support for a model in which dynamic interactions among these receptors mediate Wnt-3a activation of ATF2 signaling. 


\section{INTRODUCTION}

Wnt proteins play roles in many cellular and physiological processes, regulating cell proliferation, differentiation, migration and patterning during development and tissue homeostasis in adults, primarily through their effects on stem/progenitor cells $[1,2]$. In the canonical Wnt pathway, $\beta$-catenin is degraded by a protein complex containing Axin, glycogen synthase kinase-3 (GSK-3), casein kinase 1 and adenomatous polyposis coli. When a Wnt protein binds to its frizzled (FZD) and low-density lipoprotein receptor-related protein (LRP5/6) receptors, Axin and disheveled (DVL) are recruited to the membrane, leading to disruption of the degradation complex, $\beta$-catenin stabilization and entry into the nucleus and activation of T-cell factor/lymphoid enhancer factor-1 (TCF/LEF) target genes [1]. In addition, non-canonical Wht signals affect DVL and other intracellular components to activate the planar cell polarity (PCP) and Wnt-Calcium pathways $[3,4]$. These signals lead to a wide range of downstream effects, including changes in the cytoskeleton, calcium release from intracellular storage sites, activation of protein kinases [5-7] and subsequent activation of transcription factors, notably members of the AP-1 family $[8,9]$.

Canonical and non-canonical Wnt receptors (FZD1-10 and VANGL1/2) and coreceptors (ROR1/2, RYK, PTK7, LRP4-6) form heterodimers, and the nature of the signal transduced is believed to be determined by the repertoire of receptor complexes and Wnt ligands expressed [10]. Competition among Wnt proteins for common receptors can give rise to different outcomes. For example, Wnt-5a, which preferentially activates PCP signaling, competes for and inhibits binding of Wnt-3a to FZD2, thereby suppressing $\beta$-catenin-dependent signaling [14].

Although several Wnts preferentially activate either $\beta$-catenin-dependent (Wnt- 
$1 / 3 a$ ) or $\beta$-catenin-independent (Wnt-5a/11) signals, the activity of a particular Wht often depends on cellular context and receptor availability (LRPs versus RORs, for example), and thus Wnts cannot be rigorously subdivided according to the signaling pathway they regulate $[10,11]$. Supporting this idea, we recently reported that Wnt-3a, which is generally considered to be a canonical Wnt [12], promotes early differentiation of human neural stem cells (NSCs) by activating a non-canonical signal via the AP-1 family member ATF-2 [13]. In that study we showed that neuronal differentiation is accompanied by reductions in $\beta$ catenin/Tcf-dependent transcription and expression of $\beta$-catenin/Tcf target genes, and that neither activation nor inhibition of Wnt/ $\beta$-catenin signaling affects hNP cell differentiation. Moreover, addition of recombinant Wnt-3a to cells blocked for Wht secretion restores neuronal differentiation, and this rescue was shown by immunocytochemistry not to be blocked by inhibition of Wnt/ $\beta$-catenin signaling using Dkk-1, which downregulates canonical Wnt co-receptors, or by IWR-1, which promotes $\beta$-catenin degradation by stabilizing Axin. Finally, we showed that differentiation is accompanied by an increase in endogenous WNT3A expression and that silencing of WNT3A inhibits neuronal differentiation.

Wnt receptors, like Wnts, have been studied extensively. Loss-of-function studies frequently associate Wnt receptors with neural phenotypes, both in mouse and cell-based models. Targeted deletion of the genes encoding mouse Fzds 3, 6 and 9 cause defects in axon guidance and midbrain morphogenesis and increased cell death in the developing dentate gyrus [15-17]. In addition, Fzd-1 participates in the transmission of a canonical Wnt-1-mediated neuroprotective signal in dopaminergic neurons [18], while targeted deletion of Lrp6 delays dopaminergic neuron development [19]. Non-canonical Wnt receptors are also implicated in 
neuronal development. Vangl2, for example, regulates the asymmetric division of mouse SVZ progenitors [20], combined knockout of Ror1 and Ror2 results in axon branching defects [21], and Ryk knockout disrupts axon guidance [22]. Cell-based models have demonstrated that primary neuronal progenitor cell differentiation is regulated by Ror1 and Ror2 [23] and by Wnt3-induced cleavage of Ryk, whose cytoplasmic domain then plays a role in the nucleus [24]. These studies, primarily carried out using mouse models, highlight the importance of several Wnt receptors in neuronal differentiation. However, less is known about Wnt receptors that control human NSC differentiation. In this report we have used gene silencing to identify Wnt receptors required for differentiation of human embryonic stem cellderived NSCs, which we previously reported to be mediated by Wnt-3a/ATF2. We show that simultaneous silencing of two Wnt receptors is required to inhibit Wnt3a-induced neuronal differentiation, and we identify FZD4, FZD5, ROR1 and LRP6 as the key receptors involved. We further use non-neural cell lines to show that FZDs, ROR1 and LRP6 are able to colocalize on the cell surface, coimmunoprecipitate and activate ATF2-dependent transcription. Our observations are consistent with a model in which Wnt-3a promotes NSC differentiation via FZD4, FZD5, LRP6 and ROR1 receptor complexes.

\section{MATERIALS AND METHODS}

\section{Cell culture and drug treatments}

Human neural progenitor (hNP) cells (Aruna Biomedical, Athens, GA) were cultured at $37^{\circ} \mathrm{C}, 5 \% \mathrm{CO}_{2}$ in Neurobasal Medium with $1 \times \mathrm{B} 27$ Supplement, $1 \mathrm{x}$ penicillin/streptomycin (P/S) and $2 \mathrm{mM}$ L-glutamine (Life Technologies) plus 20 ng/ml basic Fibroblast Growth Factor (bFGF, Life Technologies) and $10 \mathrm{ng} / \mathrm{ml}$ 
Leukemia Inhibitory Factor (ProSpec) on Matrigel ${ }^{\mathrm{TM}}$-coated plates. Differentiation was induced by removal of bFGF from the medium. Unless stated otherwise, cells were induced to differentiate for 3 days, as described previously [13]. HEK 293 cells were cultured in high glucose DMEM with GlutaMAX ${ }^{\mathrm{TM}}-1$ (Life Technologies), 10\% FCS (First Link UK) and P/S. Wnt-C59 (100 nM) (Cellagen Technology), IWR1-endo (10 uM) (Calbiochem) or the equivalent volumes of DMSO as controls, were diluted in medium prior to use. Recombinant Wnt-3a (R\&D Systems) was used at $100 \mathrm{ng} / \mathrm{ml}$, with corresponding dilutions of vehicle (1\% CHAPS) as a control. If not stated otherwise, cells were treated for 3 days. L-M(tk-) (ATCC CCL-1.3), L Wnt-3a cells (ATCC CRL-2647) and HEK 293T cells were cultured in DMEM with 10\% FCS, P/S and $0.4 \mathrm{mg} / \mathrm{ml} \mathrm{G-418} \mathrm{(L-Wnt-3a} \mathrm{cells).}$

\section{RNA analysis}

RNA was extracted using the PureLink RNA Micro Kit (Life Technologies), according to manufacturer's instructions. RNA purity and quantity were measured on a Nanodrop spectrophotometer and 1 ug of total RNA was transcribed with MMLV-RT (Life Technologies) in the presence of RNaseOUT (Life Technologies), according to manufacturer's instructions. Real-Time PCR was performed in a ViiA7 System (Applied Biosystems) using Perfecta SYBR Green SuperMix Low Rox (Quanta). The $\Delta \Delta$ Ct quantitation method was used to determine mRNA fold changes in gene expression, with $36 B 4$ as the housekeeping gene. The primers

used are listed in Supplementary Table 1. The TaqMan® Array, Human WNT Pathway, was used as instructed by the manufacturer (Applied Biosystems).

\section{Gene reporter assays}

Cells plated in 24-well plates at $40 \%$ confluence were washed twice with 
OptiMEM (Life Technologies) to remove antibiotics and each well was transfected with 100 ng ATF-2-luciferase gene reporter, 25 ng of a constitutive Renilla luciferase reporter (pRL-tk) and $400 \mathrm{ng}$ (or $200+200 \mathrm{ng}$ ) of each receptor (pRK5/pRK5-FZD4/pRK5-FZD5, pcDNA3/pcDNA3-ROR1, pCS2/pCS2-LRP6) using Lipofectamine LTX and Plus Reagent (Life Technologies), as instructed by the supplier. After 24 h, cells were washed twice with PBS and lysed in 100 ul Passive Lysis Buffer (Promega). Luminescence was determined using the Dual-Glo Luciferase Assay System (Promega). Signaling activities were calculated as luciferase/Renilla ratio.

\section{Receptor overexpression and gene silencing}

Cells plated in $35 \mathrm{~mm}$ plates at $30 \%$ confluence were washed twice with OptiMEM to remove antibiotics and transfected with 1 ug of each vector pRK5/pRK5-FZD5-1D4, pcDNA3/pcDNA3-ROR1, pCS2/pCS2-LRP6 for a total of 2 ug per plate, using Lipofectamine LTX and Plus Reagent. After $24 \mathrm{~h}$, the transfection mix was replaced with normal medium. Cells were lysed after a further $24 \mathrm{~h}$. For gene silencing, cells plated in 12-well plates at $50 \%$ confluence were washed twice with OptiMEM to remove antibiotics and transfected using Lipofectamine RNAiMAX (Life Technologies) with 50 nM ON-TARGETplus siRNA SMARTpools (GE Healthcare) containing 4 different siRNA sequences. The siRNA transfection method was previously used by our group and achieves over $50 \%$ silencing of mRNA and protein levels (Ref 13). When more than one siRNA was transfected into the cells, each siRNA was transfected at $25 \mathrm{nM}$ with the corresponding volume of Lipofectamine RNAiMAX. In cases of double and quadruple silencing, the amounts of control siRNA were adjusted accordingly. The transfection mix was removed after $24 \mathrm{~h}$ incubation and fresh media (with or 
without treatment) were added to the cells. The siRNA sequences are available upon request.

\section{Immunoprecipitation and western blotting (WB)}

Cells were incubated with $50 \mathrm{ng} / \mathrm{ml}$ of recombinant Wnt-3a (R\&D Systems) for 10-15 min at $37^{\circ} \mathrm{C}, 5 \% \mathrm{CO}_{2}$. Cells were washed twice with PBS and total cell extracts were obtained by lysis in immunoprecipitation buffer $(1 \%$ Triton $\mathrm{X}-100$, $50 \mathrm{mM}$ Tris $\mathrm{pH}$ 8.0, $150 \mathrm{mM} \mathrm{NaCl}$ (all Sigma), cOmplete EDTA-free Protease Inhibitors (Roche) and PhosSTOP (Roche). The lysates were centrifuged for $12 \mathrm{~min}$ at $15,000 \mathrm{~g}$ and the supernatants were divided into fresh tubes on ice containing antibodies as indicated on ice overnight. They were then incubated with $15 \mathrm{ul}$ of Protein A/G agarose (Pierce or Santa Cruz) and incubated for $1 \mathrm{~h}$ on a wheel at $4^{\circ} \mathrm{C}$. The beads were subsequently washed three times with immunoprecipitation buffer and once with PBS and eluted with 15 ul of 2x Laemmli sample buffer (Sigma), and either heated at $37^{\circ} \mathrm{C}$ for $10 \mathrm{~min}$ (this temperature was used to reduce FZD aggregation) for analysis by western blotting. Proteins were separated and analyzed as described previously [13]. Antibodies used for WB are listed in Supplementary Table 2.

\section{Immunofluorescence}

Immunofluorescence in hNP cells was performed and quantified as described previously [13]. Images were taken consecutively with the same exposure time using a Leica Epifluorescence microscope. The threshold was fixed for each channel in order to select and measure the stained area for each image using ImageJ. For immunofluorescence of $\mathrm{L}$ and L-Wnt-3a cells, cells seeded on coverslips at $70 \%$ confluence were washed twice with PBS and transfected with a 
total of 500 ng plasmid DNA using lipofectamine LTX and Plus reagent. The transfection mix was removed after $3 \mathrm{~h}$ and fresh medium added. After 24 hours, coverslips were rinsed with PBS and incubated with primary antibodies on ice for 1 hour. All primary antibodies were diluted in complete medium with $20 \mathrm{mM}$ HEPES pH 7.4. Coverslips were then fixed in 4\% paraformaldehyde for 20 minutes, washed 3 times with PBS and incubated with secondary antibodies (AlexaFluor488 and AlexaFluor594, Life Technologies) diluted in PBS with 3\% BSA and $50 \mathrm{mM}$ glycine in the dark for 1 hour at room temperature. Cells were then washed 3 times with PBS and mounted using DAPI-Vectashield (Vector Labs). Cells were visualized using a Leica Confocal microscope. Image analysis and quantification were performed using ImageJ. Colocalization was quantified using Pearson's correlation coefficient, using a minimum of five representative cells from two independent experiments. The primary antibodies used for immunocytochemistry are listed in Supplementary Table 2.

\section{Statistics}

Three independent experiments were performed for each panel, unless otherwise stated. Students $t$ test or ANOVA followed by Dunnett's was applied to determine significance, as required. Error bars show SD.

\section{RESULTS}

An siRNA screen for Wht receptors involved in neuronal differentiation identifies FZD4, FZD5, ROR1 and LRP6 as potential candidates.

We previously found that WNT3A expression increased during neuronal differentiation of hNP cells and showed that blocking this increase using WNT3A 
siRNA is sufficient to inhibit differentiation. We hypothesised that the same may be true for the Wnt receptor(s) that transduce the WNT3A signal. Analysis of Wnt receptor expression in differentiating hNP cells found upregulation of FZD7 and VANGL2 ([13] and Supplementary Fig. 1). However, neuronal differnetiation was not inhibited by FZD7 or VANGL2 siRNAs [13]. This suggests that undifferentiated cells already express Wnt receptor(s) required for differentiation. We therefore silenced each of the Wnt receptors expressed by hNP cells, excluding FZD1, FZD8, FZD9, LGR6 and MUSK, since these were either present at very low levels or undetectable. None of the Wnt receptor siRNAs strongly inhibited neuronal gene expression, suggesting that there is redundancy at the receptor level. We did however observe a small increase in neuronal gene expression upon silencing of ROR2 (Fig. 1 and Supplementary Table 3). This suggests that endogenous ROR2 inhibits differentiation and is consistent with its downregulation during differentiation (Supplementary Fig. S1). We also examined the effect of silencing each receptor on the expression of the other receptors to identify potential coregulation and/or cross-silencing effects. This revealed that FZD3 silencing reduced the expression of ten other Wnt receptors by over 40\% (Fig. 1 and Supplementary Table 3). The reason for this is not known, but it prevented us from studying FZD3 further because it would make the results difficult to interpret.

Since Wnt signals are often transduced by a member of the FZD family in combination with a single-pass transmembrane receptor, we hypothesized that silencing one receptor from each class would inhibit differentiation. We therefore silenced pairs of Wnt receptors and examined the effects on neuronal gene expression. In several cases, we observed reduced neuronal gene expression (Fig. 2 and Supplementary Table 4). The siRNAs that had the strongest pairwise effects on 
neuronal gene expression were those that targeted ROR1, LRP6 and FZD5. As observed for single receptor silencing, ROR2 siRNA increased neuronal gene expression when combined with several multi-pass membrane receptor siRNAs. The effect of ROR2 siRNA was weakest when combined with FZD5 siRNA, consistent with FZD5 siRNA inhibiting differentiation. The mRNA levels of the receptors were also measured in order to provide an indication of the extent of receptor silencing (Supplementary Fig. 2 and Supplementary Table 5). Taken together, these experiments point to a requirement for LRP6, ROR1 and FZD5 in neuronal differentiation of hNP cells.

Pair-wise silencing of FZD4, FZD5, ROR1 and LRP6 inhibits neuronal differentiation of hNP cells.

In light of the findings above, we carried out further analysis of those receptors whose silencing showed the strongest trends for inhibition of neuronal gene expression. We found that combined silencing of ROR1 with FZD4 or FZD5, or, to a lesser extent with FZD2, reduced neuronal gene expression (Supplementary Fig. 3). Similarly, combined silencing of LRP6 with FZD4 or FZD5 had the strongest effect on neuronal gene expression (Supplementary Fig. 4). For FZD5 the strongest effect was observed when it was silenced with ROR1 (Supplementary Fig. 5). Given these results, we further examined the effects of silencing FZD4 and observed inhibition of neuronal gene expression upon combined silencing with ROR1 or LRP6 (Supplementary Fig. 6). Of note, in these secondary screens we observed that single silencing FZD4 and FZD5 alone, and to a lesser extent of LRP6, showed a trend for reducing neuronal gene expression (Supplementary Figs. 3-6). However, the effects were statistically significant only when two receptors were silenced 
together (Fig. 3a and c). Silencing all four receptors together reduced neuronal gene expression to a similar extent as silencing pairs of receptors (compare Figs. $3 a$ and $b)$.

To determine if the effects of Wnt receptor gene silencing observed at the level of neuronal gene expression resulted in changes in differentiation at the cellular level, experiments were repeated and the expression of neuronal markers analyzed by immunofluorescence. hNP cells transfected with control siRNA and induced to differentiate for 4 days expressed high levels of ND1 and DCX proteins (Fig. 4a), as previously observed [13]. Pair-wise silencing of FZD4 or FZD5 in combination with either ROR1 or LRP6 resulted in strong inhibition of expression of both ND1 and DCX proteins (Fig. 4a and b). Silencing all four Wnt receptors together did not further enhance this effect (Figs. 4c and d), consistent with results in Fig. 3d. Together, these findings suggest that a combination of the Wnt receptors FZD4, FZD5, ROR1 and LRP6 are important for neuronal differentiation.

We previously showed that neuronal differentiation of hNP cells is accompanied by an increase in endogenous WNT3A expression and that silencing of WNT3A inhibits neuronal differentiation [13]. We therefore hypothesized that the Wnt receptors we identified promote differentiation by transducing endogenous WNT3A signals. To determine if these receptors are required for the differentiation response to exogenously added WNT3A, silencing experiments were carried using cells treated with recombinant Wnt-3a plus Wnt-C59, which blocks secretion of endogenous Wnt proteins, and IWR-R1, which blocks Wnt/ $\beta$-catenin signaling [13]. Pair-wise silencing of Wnt receptors reduced non-canonical Wnt-3a-induced neuronal gene expression (Fig. 5), and the effect was similar to that observed in differentiating cells (Fig. 3a), indicating that silencing Wnt receptor pairs is 
sufficient to inhibit the differentiation response to exogenous Wnt-3a.

Ectopic expression of noncanonical Wht receptors activates ATF-2-dependent transcription.

In our earlier study, we showed that ATF2 levels and ATF2-dependent transcription increase during neuronal differentiation of hNP cells and that gene silencing of ATF2 inhibits differentiation [13]. We therefore hypothesized that the Wnt receptors we identified might activate ATF-2. To test this possilbity, we attempted to express Wnt receptors in hNP cells. However, this invariably resulted in cell death (results not shown), probably as a result of stress caused by high expression of transmembrane proteins. We therefore carried out experiments using HEK 293 cells, which are more resilient. Ectopic expression of ROR1, LRP6 and FZD5 increased the activity of an ATF2-dependent luciferase reporter [25] (Supplementary Fig. 7a), while FZD4 transfection showed a trend for an increase. We were concerned that the expression of adenovirus protein E1a in HEK 293 cells, which activates ATF-2 [26], might mask the Wnt response. Experiments were therefore repeated using L-M tk- (L) cells, which have lower basal ATF-2 activity and do not express endogenous Wnt-3a or ROR1/2 [27, 28]. In addition to ATF-2 luciferase, we used a CREB-dependent CRE-luciferase reporter, since the AP-1 family member CREB is activated by non-canonical Wnt-3a signaling in hNP cells [13] and by non-canonical Wnt signals in other cell types [29]. ROR1 and FZD5 increased ATF2-dependent transcription, while expression of FZD4 again showed a trend for an increase, but LRP6 had no effect (Supplementary Fig. 7b). None of the receptors affected the activity of the CRE-luciferase reporter (results not shown). Experiments were also carried out using L cells stably expressing Wnt-3a (L-Wnt-3a cells). L-Wnt-3a cells had slightly higher basal ATF-2-dependent 
transcriptional activity, compared to L cells $(\mathrm{p}=0.054)$ but ectopic expression of Wnt receptors in L-Wnt-3a cells did not further increase ATF2- or CRE-luciferase gene reporter activity (Supplementary Fig. $7 \mathrm{~b}$ and results not shown). In conclusion, the gene reporter assay results indicate that ROR1 and FZD5 and, to some extent FZD4, are able to activate ATF2-dependent transcription in HEK 293 and $L$ cells.

Colocalization and co-immunoprecipitation of ROR1, FZD4/5 and LRP6 in transfected cells.

Our studies in hNP cells highlight four Wnt receptors as playing an important role in the non-canonical response to Wnt-3a, but it is not clear whether they function together to activate the same signal or pairwise to activate different signals. To try to distinguish these possibilities, we carried out immunocytochemistry experiments to look for evidence of complex formation. These experiments were carried out in L cells, since ectopic expression of the receptors in hNP cells resulted in cell death and we were unable to detect the endogenous receptors. Immunocytochemistry analysis revealed cell-surface colocalization of transfected LRP6 and FZD4 in L cells and in L-Wnt-3a cells (Fig. 6b), consistent with previous studies examining the association of LRP6 with FZD family members [30-32]. Quantitative analysis of the immunofluorescence data indicated that LRP6 and FZD4 showed strong co-localization in L cells and that this was slightly lower in L-Wnt-3a cells. Examination of cells co-transfected with ROR1 and FZD4 showed cell-surface co-localization of these receptors also (Fig. 6b), as has been reported previously for ROR2 and FZD family members [11,33]. Quantitation of the images indicated that the extent of co-localization of ROR1 and FZD4 was similar in L and L-Wnt-3a cells and in the same range as the co- 
localization of LRP6 and FZD4 in L cells. Finally, we examined ROR1 and LRP6, whose co-localization, as far as we are aware, has not been reported previously. ROR1 and LRP6 partially co-localized in L cells and in L-Wnt-3a cells (Fig. 6c). The extent of ROR1/LRP6 co-localization in L cells was slightly lower than those for R0R1/FZD4 and LRP6/FZD4. Together, these results indicate that ROR1, FZD4 and LRP6 have the potential to interact at the cell surface and they further suggest that these interactions differ to some extent in L-Wnt-3a cells. Unfortunately, we were unable to carry out similar analyses for FZD5 using the antibodies available.

To determine to what extent the four Wnt receptors were present in the same complexes, we immunoprecipitated Wnt receptors from HEK 293 cells transfected with pairs of receptors and treated with vehicle or purified Wnt-3a and probed them by western blotting. The results indicated that ROR1 could be coimmunoprecipitated with FZD4 and with LRP6 (Fig. 7a; we were unable to carry out a similar analysis for FZD5 using the antibodies available). LRP6 could also coimmunoprecipitated with FZD4 and with FZD5 (Fig. 7b), as previously reported [34]. FZD4 and FZD5 migrated as monomers and multimers on gels, as has been observed for other members of the FZD family [35]. Treatment of cells with Wnt3a did not significantly affect the association between LRP6 and ROR1 (Fig. 7a), but it reduced LRP6 association with FZD5 and increased its association with FZD4 multimers (Fig. 7b). In summary, these results indicate that ROR1, LRP6 and FZD4/5 are able to form complexes with one another and that Wnt-3a treatment results in a change in the interactions among LRP6 and FZD4 and FZD5.

\section{DISCUSSION}

An siRNA-based screen for Wnt receptors involved in neuronal differentiation 
We previously reported that Wnt secretion, Wnt-3a in particular, is essential for neuronal differentiation of hNP cells, but the identities of the receptors involved were not known [13]. Here we show that silencing of single Wnt receptors in hNP cells is insufficient to inhibit neuronal differentiation (Fig. 1). This is in contrast to what we observed upon silencing WNT3A or its target ATF2 [13], and suggests that there is redundancy at the receptor level. However, in our secondary screens silencing of FZD4 or FZD5 did show a trend for reducing neuronal gene expression, and we subsequently confirmed that these receptors were required for differentiation. Our silencing screen also identified ROR2 as a negative regulator of neuronal differentiation: ROR2 expression levels were reduced during differentiation and ROR2 silencing increased neuronal gene expression. These results are somewhat unexpected, since that ROR2 has been associated with processes involving neuronal maturation, such as neurite extension and synapse formation [36, 37]. One possibility is that ROR2 represses early neural differentiation and is upregulated at a later stage of differentiation, where it may play a role in neuron maturation.

Pair-wise silencing of Wht receptors identifies FZD4, FZD5, ROR1 and LRP6 as mediators of neuronal differentiation.

The two-by-two receptor silencing screen and the subsequent validation experiments indicated that pair-wise silencing of ROR1 with FZD4 or FZD5 and LRP6 with FZD4 or FZD5 is sufficient to inhibit neuronal differentiation (Supplementary Figure 8). A requirement for LRP6 was somewhat unexpected, since LRP6 is best known for transducing canonical Wnt signals. Moreover, we previously found that treatment of hNP cells with Dkk-1, which binds and internalizes LRP5/6, does not affect neuronal gene expression, and that combined 
treatment of cells with Wnt-3a and Dkk-1 actually enhances differentiation [13]. LRP6 appears to be dispensable for differentiation in cells treated with exogenous Wnt-3a, so its role might be to serve as a co-receptor for other Wnts, such as Wnt7a or Wnt-7b, which play a role at later stages of neuronal differentiation $[38,39]$. Indeed, Wnt-7a has been reported to bind to a LRP6-FZD5 receptor complex in PC12 cells [34]. In addition, LRP6 may serve as a co-receptor for ROR1, since ROR1 can bind to Wnt-3, albeit weakly [40]. The cell-surface co-localization and coimmunoprecipitation of ROR1 and LRP6 that we observed in transfected cells (Fig. 6) support this possibility. Unlike LRP6, ROR1 has a CRD and, according to the Wnt-8-FZD8 CRD structure and reports that ROR2 binds to Wnts [8, 41], ROR1 could transduce the non-canonical Wnt-3a signal.

In the first two-by-two screen, silencing of either ROR1 or LRP6 in combination with silencing of a FZD $(2,4$ or 5$)$ reduced neuronal marker gene expression. It is likley, therefore, that there is some redundancy among the FZD receptors. This may not be surprising, given that the lipid anchor site is conserved in FZD family members and their specificity is mediated by variations at a second site [41]. Thus, the specificity of the binding interaction might be determined by the availability of co-receptors and soluble factors, not only the Wnt ligands themselves but also sFRP family members [42]. Interestingly, Wnt-3a treatment of transfected HEK 293 cells favored association of LRP6 with FZD4 over FZD5, suggesting that these FZDs could play different roles in some settings. As these experiments were carried out in non-neural cells, the results are only suggestive of what these Wnt receptors may be capable of in hNP cells. We attempted to carry out experiments using hNP cells transfected with Wnt receptor expression constructs but this resulted in cell death, probably as a result of stress imposed on the ER/Golgi from 
high levels of transmembrane protein expression. An alternative approach would be to study the endogenous Wnt receptors. However, the Wnt receptor antibodies available to us were not sensitive enough to do this.

\section{Activation of ATF2-dependent transcription by Wht receptors}

Our earlier study linked non-canonical Wnt-3a signaling to activation of JNK and ATF2 in NSCs. Since ROR1 can activate JNK/CREB in cancer cells $[43,44]$ and ROR2 has been reported to activate ATF2 [45], it seemed plausible that ROR1 plays a role in the same signaling cascade. Indeed, ectopic expression of ROR1 increased ATF2dependent transcriptional activity in HEK 293 and L cells (Supplementary Fig. 7a and b). FZD5 also activated this reporter, consistent with a previous report showing that FZD5 plays a role in neuronal maturation via JNK [46]. However, ATF2-dependent transcriptional activity was not further increased by ectopic expression of FZD4/5, LRP6 or ROR1 in L-Wnt-3a cells (Supplementary Fig. 7b), or by treatment of HEK 293 cells with Wnt-3a (results not shown). One possibility is that the high $\beta$-catenin/Tcf signaling activity in L-Wnt-3a and Wnt-3a-treated HEK 293 cells is not compatible with Wnt-3a activation of ATF-2. In conclusion, while the Wnt receptors we have identified can activate ATF2-dependent transcription, further studies using neural cells will be required to determine if this is liganddependent.

Finally, our results do not exclude the possibility that Wnts other than Wnt-3a promote neuronal differentiation via FZD4/5, LRP6 and ROR1. Wnt-7a, for example, is also upregulated upon differentiation of hNP cells [13] and has been reported to associate with FZD5 during synaptogenesis [47]. Consequently, the Wnt receptors that are required for NSC differentiation may play distinct roles in vivo: first in response to non-canonical Wnt-3a during early neuronal induction 
and then in the maturation of the new-born neurons in response to Wnt-7a.

\section{Acknowledgments}

This work was facilitated by funding from the Spanish Ministry of Education and Science (SAF2014-51966-R, IJCI-2014-21255) and the Government of the Autonomous Community of the Basque Country Departments of Education, Industry, Tourism and Trade (Elkartek) and Innovation Technology. We thank Maria Vivanco for critical reading of the manuscript. 


\section{Figure legends}

Fig. 1. Effects of silencing single Wnt receptors on neuronal gene expression in hNP cells Cells were transfected $50 \mathrm{nM}$ for each siRNA and induced to differentiate for $72 \mathrm{~h}$. The levels of Wnt receptor and neuronal gene (DCX, ND1 and NEUROG1) mRNAs, relative to 36B4, were measured by qPCR. (a, b) Heatmaps represent mRNA levels, relative to cells transfected with control siRNA (siCTRL). Each row represents samples transfected with the siRNA indicated on the left and each column represents the expression of the gene indicated at the top. The scale to the right indicates the extent of up-/down-regulation from 0.1 (equivalent to a 10 -fold reduction in expression) to 2 (a 2-fold increase in expression). Graph shows relative expression of neuronal genes, error bars show standard error of the mean (SEM), $\mathrm{n}=2$.

\section{Fig. 2. Effects of pair-wise silencing of Wnt receptors on neuronal gene} expression in hNP cells Cells were transfected with $25 \mathrm{nM}$ of each siRNA and induced to differentiate for $72 \mathrm{~h}$. The mRNA levels of DCX (a), NGN1 (b) and ND1 (c) were measured by qPCR, relative to 36B4. Heatmaps represent mRNA levels, relative to cells transfected with control siRNA (siCTRL). Each sample was silenced for two genes as indicated: a multi-pass membrane receptor (top) and a singlepass receptor (left), and relative expression of each neuronal gene is shown using color-coding using the scale (bottom), which indicates the extent of up-/downregulation from 0.1 (equivalent to a 10 -fold reduction in expression) to 2 (a 2 -fold increase in expression). Graphs show relative expression of the neuronal genes silenced for each pair of Wnt receptor genes; error bars show SEM, $\mathrm{n}=2$. 
Fig. 3. Effects of combined silencing of Wnt receptors on neuronal gene expression (a-d) Cells were transfected with $25 \mathrm{nM}$ of each siRNA (F4: FZD4, F5: FZD5 and R1: ROR1), either pairwise (a, c), all four receptor siRNAs together (b, d) or the same total amount of control (CTRL) siRNA (a-d), and induced to differentiate for 72 h. mRNA expression of each neuronal and Wnt receptor gene was measured by qPCR, relative to 36B4; * $\mathrm{p}<0.05$ by ANOVA.

Fig. 4. Effects of combined silencing of Wnt receptors on neuronal differentiation (a, c) Immunofluorescence of cells transfected with $25 \mathrm{nM}$ of each siRNA (F4: FZD4, F5: FZD5 and R1: ROR1) and induced to undergo neuronal differentiation for 4 days. (b, d) Quantification of neuronal marker expression obtained using ImageJ analysis of immunofluorescence signal relative to cells transfected with control siRNA (siCTRL); (b) * $\mathrm{p}<0.05$ by ANOVA, (d) * p $<0.05$ by t test; scale bars 20 um.

Fig. 5. Pair-wise silencing of FZD4, FZD5, ROR1 and LRP6 blocks noncanonical Wnt-3a-dependent neuronal gene expression. (a, b) mRNA expression of the indicated neuronal genes and Wnt receptors in differentiating cells silenced for $48 \mathrm{~h}$ using $25 \mathrm{nM}$ siRNA for each receptor (F4: FZD4, F5: FZD5 and R1: ROR1) or control (CTRL) siRNA and treated with $100 \mathrm{nM}$ Wnt-C59, 100 ng/ml Wnt-3a and $10 \mathrm{uM}$ IWR-R1 for a further $48 \mathrm{~h} ;{ }^{*} \mathrm{p}<0.05$ by ANOVA.

Fig. 6. Analysis of Wnt receptor co-localization in $L$ and $L-W n t-3 a$ cells $L$ and L-Wnt-3a cells were transfected with FZD4 and LRP6 (a), FZD4 and ROR1 (b) or LRP6 and ROR1 (c), fixed and then analysed using immunocytochemistry to detect the transfected receptors. Pearson's correlation coefficients (mean $-/+$ s.d.) for the indicated receptor pairs are indicated on the right; scale bars $10 \mathrm{um}$. 


\section{Fig. 7. Analysis of Wnt receptor co-immunoprecipitation in HEK 293 cells}

Immunoprecipitates from extracts of HEK 293 cells transfected with the indicated pairs of Wnt receptors and treated with vehicle (CHAPS, Ch) or $50 \mathrm{ng} / \mathrm{ml}$ recombinant Wnt-3a (W3a) for 10 minutes prior to lysis were probed by western blotting as indicated; (a) control (C) and ROR1 (R1) mouse monoclonal antibody immunoprecipitates were probed for VSVG-tagged LRP6 using rabbit anti-VSVG (upper left) and FZD4 using rat anti-FZD4 (upper right); blots were then re-probed using rabbit anti-ROR1 (lower panels), the positions of LRP6, FZD4 and ROR1 are

indicated by arrowheads; (b) left: control IgG (C) and LRP6 (VSV-G, L6) rabbit antibody immunoprecipitates were probed for epitope-tagged FZD4 and FZD5 (1D4) (upper) and then reprobed for LRP6 (mouse anti-LRP5/6), right: cell extracts were probed using 1D4 and VSV-G antibodies (C, cells not transfected with LRP6), arrowheads indicate the positions of LRP6, ROR1 and FZD4 and FZD5 monomers and stars indicate the approximate positions of FZD4 and FZD5 multimers.

\section{Supplementary Fig. 1. Reduction in ROR2 and increase in VANGL2 mRNA and protein levels during hNP cell differentiation.}

(a) ROR2 and VANGL2 mRNA expression, as determined by q-PCR, in hNP cells indcued to differentiate for up to 5 days. (b) Western blots to detect the ROR2 and VANGL2 proteins in extracts of cells induced to differentiate for up to 5 days.

Supplementary Fig. 2. Extent of silencing of each receptor in the two-by-two siRNA screen $(\mathrm{a}, \mathrm{b})$ Cells were transfected $25 \mathrm{nM}$ for each siRNA and induced to differentiate for 72 h. mRNA expression of each receptor gene was measured by qPCR, relative to 36B4. Heatmaps represent mRNA levels, relative to cells 
transfected with control siRNA (siCTRL). Each row represents samples transfected with the siRNAs indicated on the left at the top, with the gene being measured shown at the bottom. The scale to the right indicates the extent of down-regulation from 0.1 (equivalent to a 10 -fold reduction in expression) to $1 ; \mathrm{n}=2$.

Supplementary Fig. 3. Secondary screen: ROR1 silencing (a, b) mRNA expression of the indicated genes in cells treated as in Fig. 2. Expression levels are normalized to those in similarly treated untransfected cells.

Supplementary Fig. 4. Secondary screen: LRP6 silencing (a, b) mRNA expression of the indicated genes in cells treated as in Fig. 2. Expression levels are normalized to those in similarly treated untransfected cells.

Supplementary Fig. 5. Secondary screen: FZD5 silencing (a, b) mRNA expression of the indicated genes in cells treated as in Fig. 2. Expression levels are normalized to those in similarly treated untransfected cells.

Supplementary Fig. 6. Secondary screen: FZD4 silencing (a, b) mRNA expression of the indicated genes in cells treated as in Fig. 2. Expression levels are normalized to those in similarly treated untransfected cells.

Supplementary Fig. 7. Effects of Wnt receptor transfection on ATF2dependent gene reporter activity. Gene reporter assays from cells transfected for $24 \mathrm{~h}$ with the indicated plasmids and ATF2-luciferase and Renilla gene reporters (V, empty vector, F4, FZD4; F5, FZD5; R1, ROR1; L6, LRP6). (a) HEK 293 cells; graph shows luciferase/renilla values normalized to values obtained from cells transfected with the respective empty vectors (V); n=3, ${ }^{*} p<0.05$. (b) L and L- 
Wnt-3a cells; graph shows luciferase/renilla ratios normalized to V in L cells; error bars show $\mathrm{SD}, \mathrm{n}=3-6,{ }^{*} \mathrm{p}<0.05$ compared to $\mathrm{V}$.

Supplementary Fig. 8. Working model for Wnt signals involved in the differentiation of hNP cells. Top Wnt-3a induces neural differentiation in hNP cells by activating AP-1 transcription factors, including ATF2, which is essential for the differentiation response (Bengoa et al., Stem Cells, 2014). However, the receptors that transduce the Wnt-3a signal at the membrane previous we not known. Bottom A gene silencing screen for Wnt receptors involved in neuronal differentiation highlighted a unique role for ROR2 in preventing differentiation and revealed a requirement for pairwise silencing of ROR1, FZD4, FZD5 and LRP6 to block Wnt-3a-induced neuronal differentiation. Studies in non-neural cells indicate that ROR1, FZD4, FZD5 and LRP6 co-localize at the cell surface, coimmunopreciptate and activate ATF-2-dependent transcription; NUC: nucleus. 


\section{References}

1. MacDonald BT, Tamai K, He X (2009) Wnt/beta-catenin signaling: components, mechanisms, and diseases. Dev Cell 17:9-26. doi: 10.1016/j.devcel.2009.06.016

2. Clevers H, Nusse R (2012) Wnt/ $\beta$-Catenin Signaling and Disease. Cell 149:1192-1205. doi: 10.1016/j.cell.2012.05.012

3. Van Amerongen R, Nusse R (2009) Towards an integrated view of Wnt signaling in development. Development 136:3205-3214.

4. Gómez-Orte E, Sáenz-Narciso B, Moreno S, Cabello J (2013) Multiple functions of the noncanonical Wnt pathway. Trends Genet 29:545-53. doi: 10.1016/j.tig.2013.06.003

5. Kohn AD, Moon RT (2005) Wnt and calcium signaling: beta-cateninindependent pathways. Cell Calcium 38:439-446.

6. Antara D (2011) Wnt / Ca2+ signaling pathway : a brief overview. Acta Biochim Biophys Sin (Shanghai) 43:745-756. doi: 10.1093/abbs/gmr079

7. Schlessinger K, Hall A, Tolwinski N (2009) Wnt signaling pathways meet Rho GTPases. Genes Dev 23:265-77. doi: 10.1101/gad.1760809

8. Schambony A, Wedlich D (2007) Wnt-5A/Ror2 regulate expression of XPAPC through an alternative noncanonical signaling pathway. Dev Cell 12:779-92. doi: 10.1016/j.devcel.2007.02.016

9. Lopez-Bergami P, Lau E, Ronai Z (2010) Emerging roles of ATF2 and the dynamic AP1 network in cancer. Nat Rev Cancer 10:65-76.

10. Niehrs C (2012) The complex world of WNT receptor signalling. Nat Rev Mol Cell Biol 13:767-79. doi: 10.1038/nrm3470

11. Grumolato L, Liu G, Mong P, et al (2010) Canonical and noncanonical Wnts use a common mechanism to activate completely unrelated coreceptors. Genes Dev.

12. Xavier CP, Melikova M, Chuman Y, et al (2014) Secreted Frizzled-related protein potentiation versus inhibition of Wnt3a/ $\beta$-catenin signaling. Cell Signal 26:94-101. doi: 10.1016/j.cellsig.2013.09.016

13. Bengoa-Vergniory N, Gorroño-Etxebarria I, González Salazar I, Kypta RM (2014) A switch from canonical to noncanonical Wnt signaling mediates early differentiation of human neural stem cells. Stem Cells 32:3196-3208. doi: $10.1002 /$ stem.1807

14. Sato A, Yamamoto H, Sakane H, et al (2010) Wnt5a regulates distinct signalling pathways by binding to Frizzled2. EMBO J 29:41-54. doi: 10.1038/emboj.2009.322

15. Wang Y, Thekdi N, Smallwood PM, et al (2002) Frizzled-3 is required for the development of major fiber tracts in the rostral CNS. J Neurosci 22:8563-73.

16. Stuebner S, Faus-Kessler T, Fischer T, et al (2010) Fzd3 and Fzd6 deficiency results in a severe midbrain morphogenesis defect. Dev Dyn 239:246-60. 
doi: $10.1002 /$ dvdy.22127

17. Zhao C, Avilés C, Abel R a, et al (2005) Hippocampal and visuospatial learning defects in mice with a deletion of frizzled 9, a gene in the Williams syndrome deletion interval. Development 132:2917-27. doi: 10.1242/dev.01871

18. L'Episcopo F, Tirolo C, Nuccio T, et al (2010) Glia as a Turning Point in the Therapeutic Strategy of Parkinsons Disease. CNS \&Neurological Disord Drug targets 9:349-372.

19. Castelo-Branco G, Andersson ER, Minina E, et al (2010) Delayed dopaminergic neuron differentiation in Lrp6 mutant mice. Dev Dyn 239:211-21. doi: 10.1002/dvdy.22094

20. Delaunay D, Cortay V, Patti D, et al (2014) Mitotic spindle asymmetry: a Wnt/PCP-regulated mechanism generating asymmetrical division in cortical precursors. Cell Rep 6:400-14. doi: 10.1016/j.celrep.2013.12.026

21. Ho H-YH, Susman MW, Bikoff JB, et al (2012) Wnt5a-Ror-Dishevelled signaling constitutes a core developmental pathway that controls tissue morphogenesis. Proc Natl Acad Sci 109:4044-51. doi: 10.1073/pnas.1200421109

22. Lu W, Yamamoto V, Ortega B, Baltimore D (2004) Mammalian Ryk is a Wnt coreceptor required for stimulation of neurite outgrowth. Cell 119:97-108. doi: 10.1016/j.cell.2004.09.019

23. Endo M, Doi R, Nishita M, Minami Y (2011) Ror family receptor tyrosine kinases regulate the maintenance of neural progenitor cells in the developing neocortex. J Cell Sci 125:2017-2029. doi: 10.1242/jcs.097782

24. Lyu J, Yamamoto V, Lu W (2008) Cleavage of the Wnt receptor Ryk regulates neuronal differentiation during cortical neurogenesis. Dev Cell 15:773-80. doi: 10.1016/j.devcel.2008.10.004

25. Ohkawara B, Niehrs C (2011) An ATF2-based luciferase reporter to monitor non-canonical Wnt signaling in Xenopus embryos. Dev Dyn 240:188-194.

26. Li X, Green MR (1996) Intramolecular inhibition of activating transcription factor-2 function by its DNA-binding domain. Genes Dev 10:517-527.

27. Shibamoto S, Higano K, Takada R, et al (1998) Cytoskeletal reorganization by soluble Wnt-3a protein signalling. Genes to Cells 3:659-670. doi: 10.1046/j.1365-2443.1998.00221.x

28. Nishita M, Itsukushima S, Nomachi A, et al (2010) Ror2/Frizzled complex mediates Wnt5a-induced AP-1 activation by regulating Dishevelled polymerization. Mol Cell Biol 30:3610-9. doi: 10.1128/MCB.00177-10

29. Chen AE, Ginty DD, Fan C-M (2005) Protein kinase A signalling via CREB controls myogenesis induced by Wnt proteins. Nature 433:317-22. doi: 10.1038 /nature03126

30. Bilic J, Huang Y-L, Davidson G, et al (2007) Wnt induces LRP6 signalosomes and promotes dishevelled-dependent LRP6 phosphorylation. Science (80- ) 316:1619-1622. doi: 10.1126/science.1137065

31. Yamamoto H, Komekado H, Kikuchi A (2006) Caveolin Is Necessary for Wnt- 
3a-Dependent Internalization of LRP6 and Accumulation of ??-Catenin. Dev Cell 11:213-223. doi: 10.1016/j.devcel.2006.07.003

32. Liang J, Fu Y, Cruciat C-M, et al (2011) Transmembrane protein 198 promotes LRP6 phosphorylation and Wnt signaling activation. Mol Cell Biol 31:2577-90. doi: 10.1128/MCB.05103-11

33. Oishi I, Suzuki H, Onishi N, et al (2003) The receptor tyrosine kinase Ror2 is involved in non-canonical Wnt5a/JNK signalling pathway. Genes to Cells 8:645-654. doi: 10.1046/j.1365-2443.2003.00662.x

34. Caricasole A, Ferraro T, Iacovelli L, et al (2003) Functional characterization of WNT7A signaling in PC12 cells: Interaction with a FZD5-LRP6 receptor complex and modulation by Dickkopf proteins. J Biol Chem 278:3702437031. doi: 10.1074/jbc.M300191200

35. Guo H, Nagy T, Pierce M (2014) Post-translational glycoprotein modifications regulate colon cancer stem cells and colon adenoma progression in Apcmin/+ mice through altered Wnt receptor signaling. J Biol Chem 289:31534-31549. doi: 10.1074/jbc.M114.602680

36. Paganoni S, Ferreira A (2005) Neurite extension in central neurons: a novel role for the receptor tyrosine kinases Ror1 and Ror2. J Cell Sci 118:433-46. doi: $10.1242 /$ jcs.01622

37. Paganoni S, Bernstein J, Ferreira a (2010) Ror1-Ror2 complexes modulate synapse formation in hippocampal neurons. Neuroscience 165:1261-74. doi: 10.1016/j.neuroscience.2009.11.056

38. Ciani L, Boyle KA, Dickins E, et al (2011) Wnt7a signaling promotes dendritic spine growth and synaptic strength through $\mathrm{Ca} 2+/$ Calmodulin-dependent protein kinase II. Proc. Natl. Acad. Sci.

39. Rosso SB, Sussman D, Wynshaw-Boris A, Salinas PC (2005) Wnt signaling through Dishevelled, Rac and JNK regulates dendritic development. Nat Neurosci 8:34-42. doi: 10.1038/nn1374

40. Billiard J, Way DS, Seestaller-Wehr LM, et al (2005) The orphan receptor tyrosine kinase Ror2 modulates canonical Wnt signaling in osteoblastic cells. Mol Endocrinol 19:90-101. doi: 10.1210/me.2004-0153

41. Janda CY, Waghray D, Levin AM, et al (2012) Structural basis of Wnt recognition by Frizzled. Science (80- ) 337:59-64. doi: 10.1126/science.1222879

42. Kawano Y, Kypta R (2003) Secreted antagonists of the Wnt signalling pathway. J Cell Sci 116:2627-2634. doi: 10.1242/jcs.00623

43. Hojjat-Farsangi M, Moshfegh A, Daneshmanesh AH, et al (2014) The receptor tyrosine kinase ROR1 - An oncofetal antigen for targeted cancer therapy. Semin Cancer Biol 1-11. doi: 10.1016/j.semcancer.2014.07.005

44. Zhang S, Chen L, Cui B, et al (2012) ROR1 is expressed in human breast cancer and associated with enhanced tumor-cell growth. PLoS One 7:e31127. doi: 10.1371/journal.pone.0031127

45. Yamagata K, Li X, Ikegaki S, et al (2012) Dissection of Wnt5a-Ror2 signaling leading to matrix metalloproteinase (MMP-13) expression. J Biol Chem 
287:1588-1599. doi: 10.1074/jbc.M111.315127

46. Slater PG, Ramirez VT, Gonzalez-Billault C, et al (2013) Frizzled-5 receptor is involved in neuronal polarity and morphogenesis of hippocampal neurons. PLoS One 8:e78892. doi: 10.1371/journal.pone.0078892

47. Sahores M, Gibb A, Salinas PC (2010) Frizzled-5, a receptor for the synaptic organizer Wnt7a, regulates activity-mediated synaptogenesis. Development 137:2215-2225. doi: 10.1242/dev.046722 
Anetser Kit 1
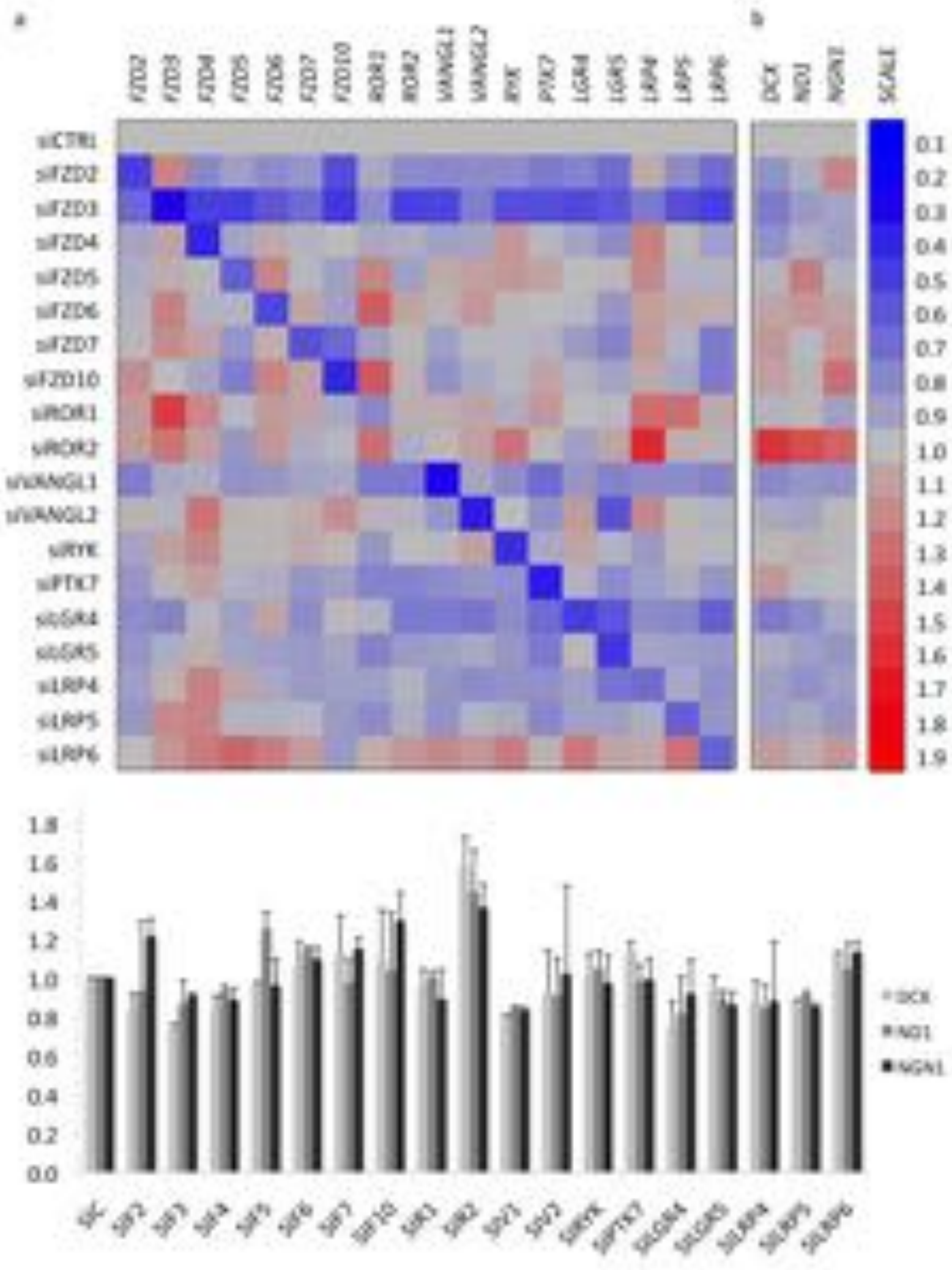
Eecostalez
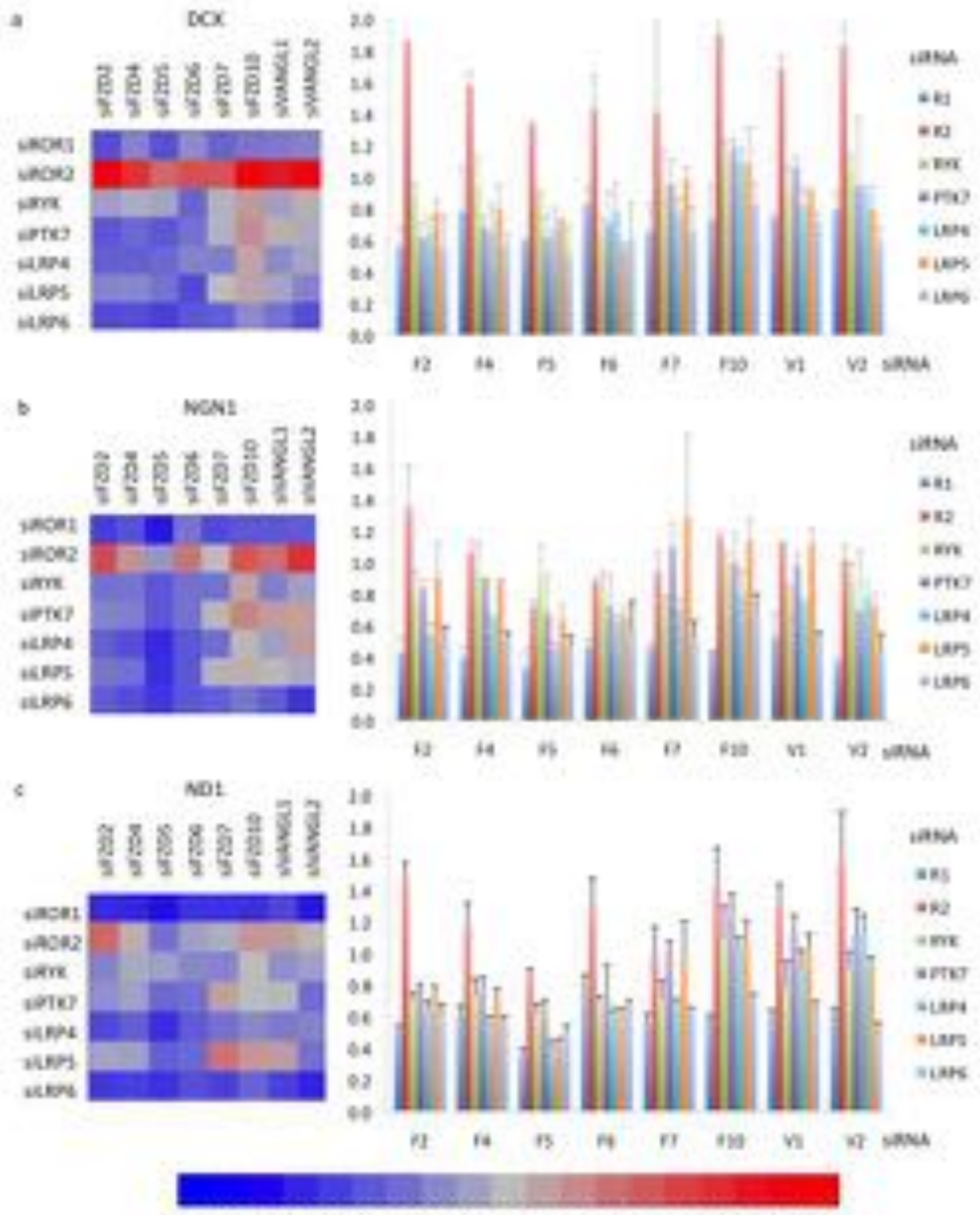

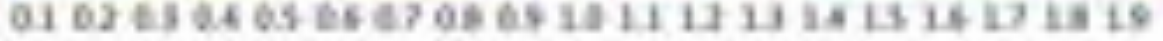




\section{Buneas at al, $\mathrm{Fe}$ l.}

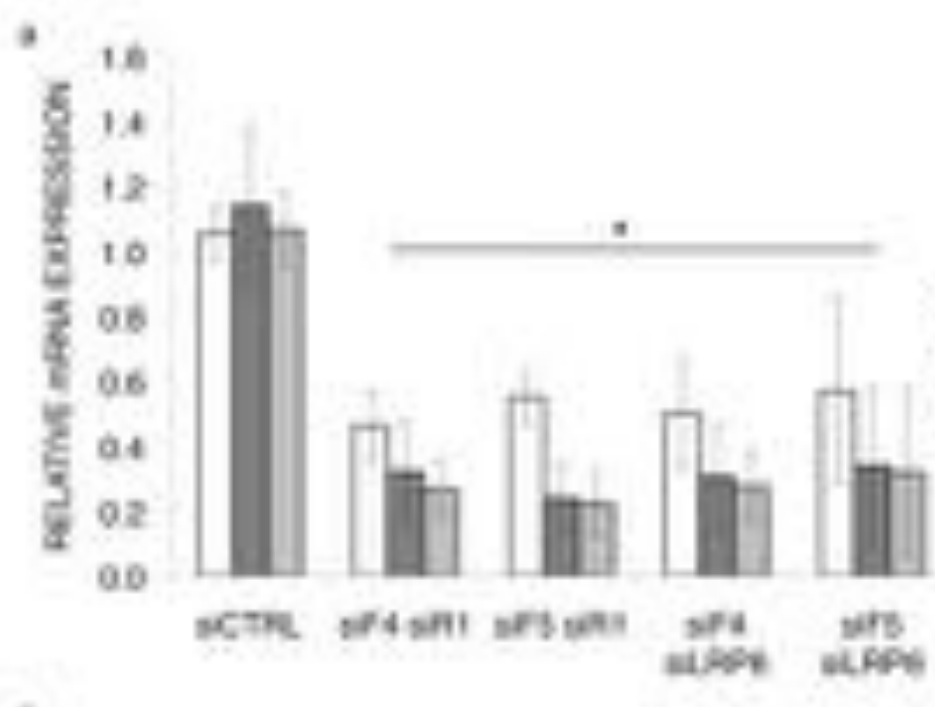

$b$
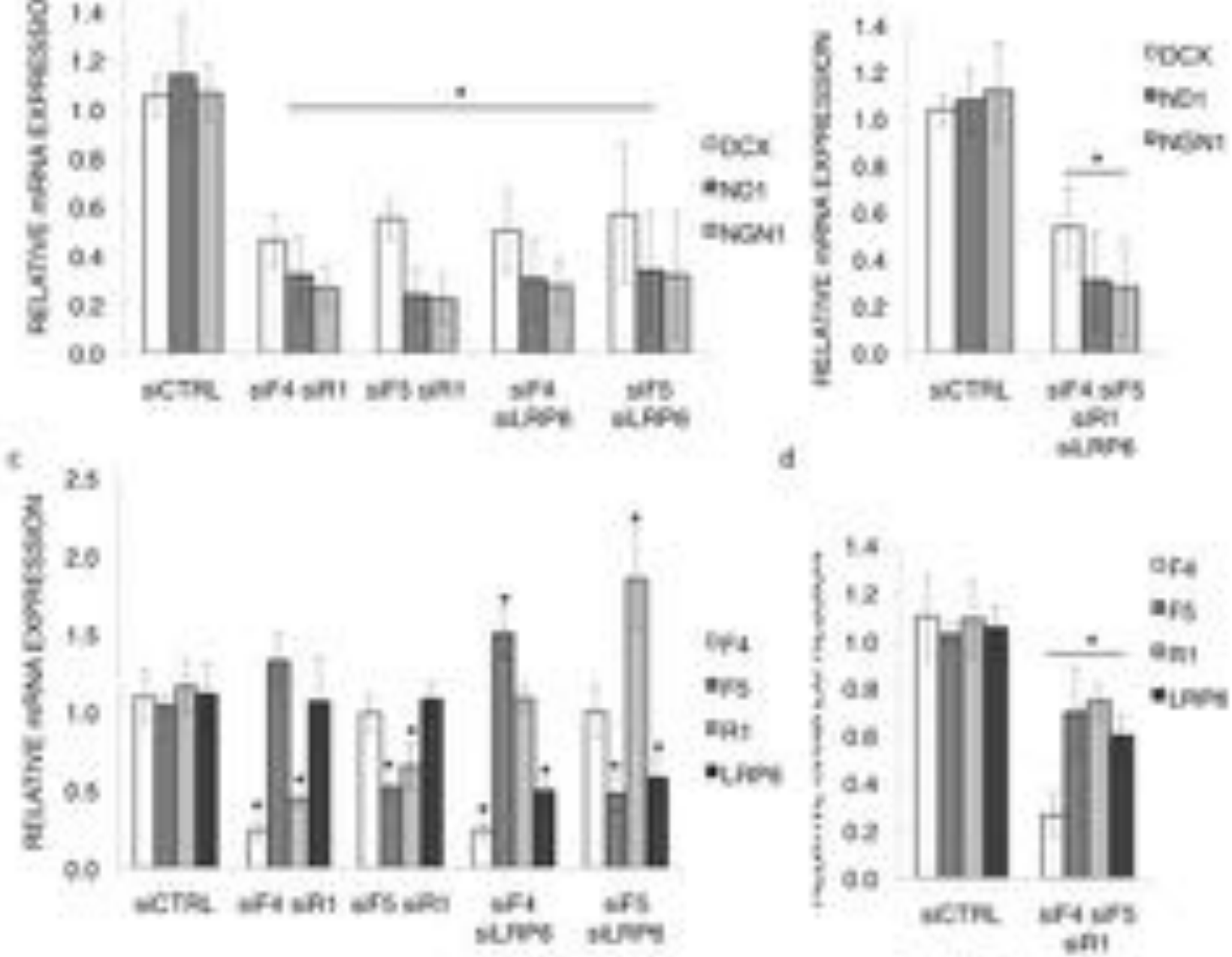

d

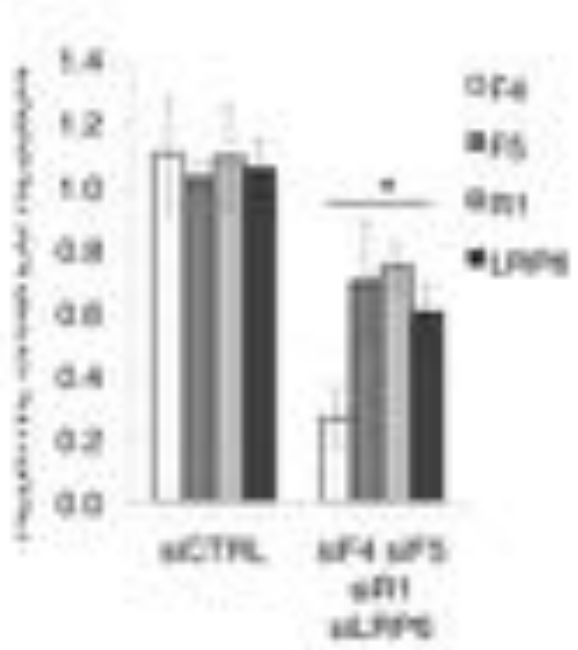


Bergar at al, fild

*
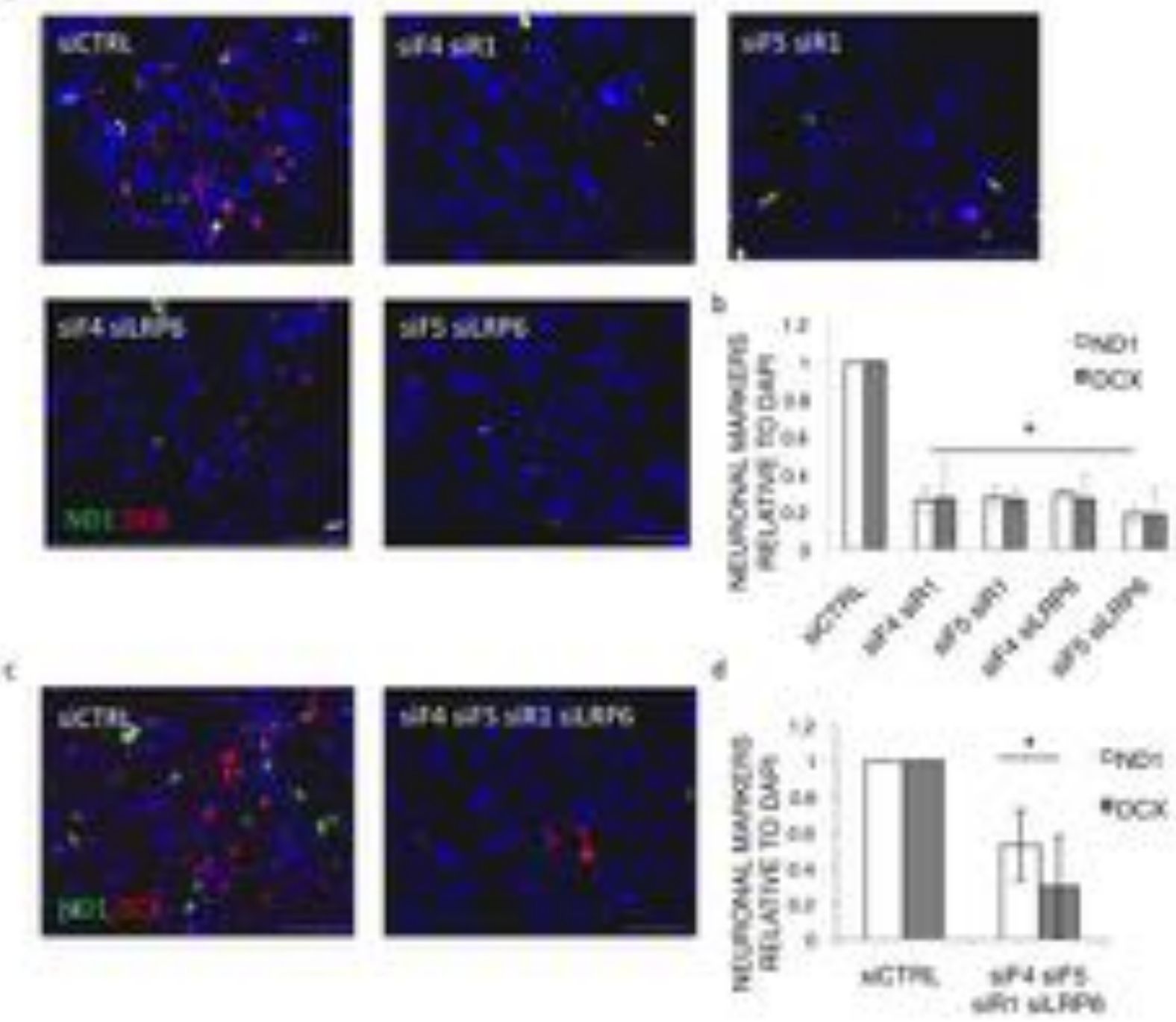


\section{feveot it 2. Fes.}

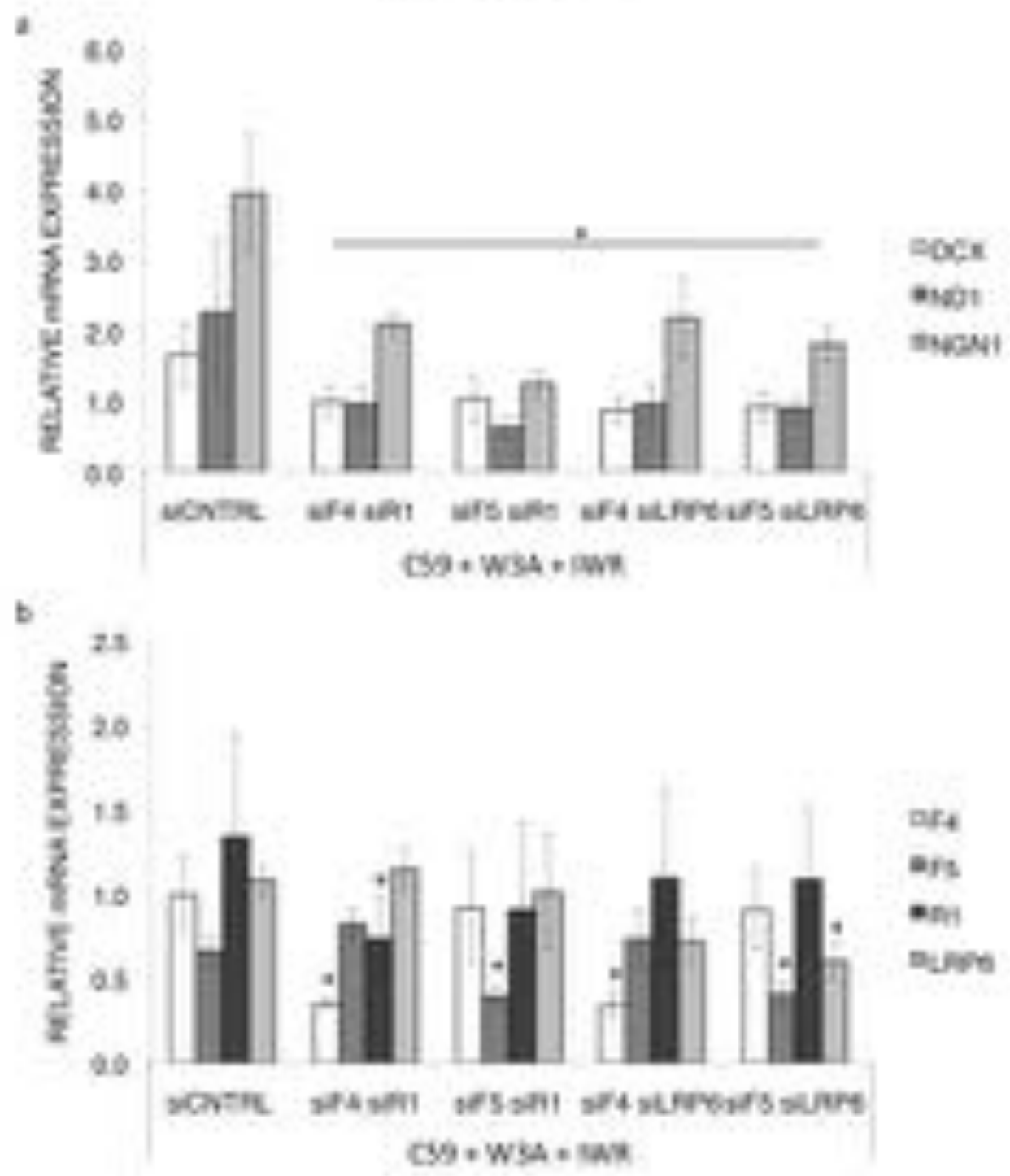


anese te al, Fe 6

3

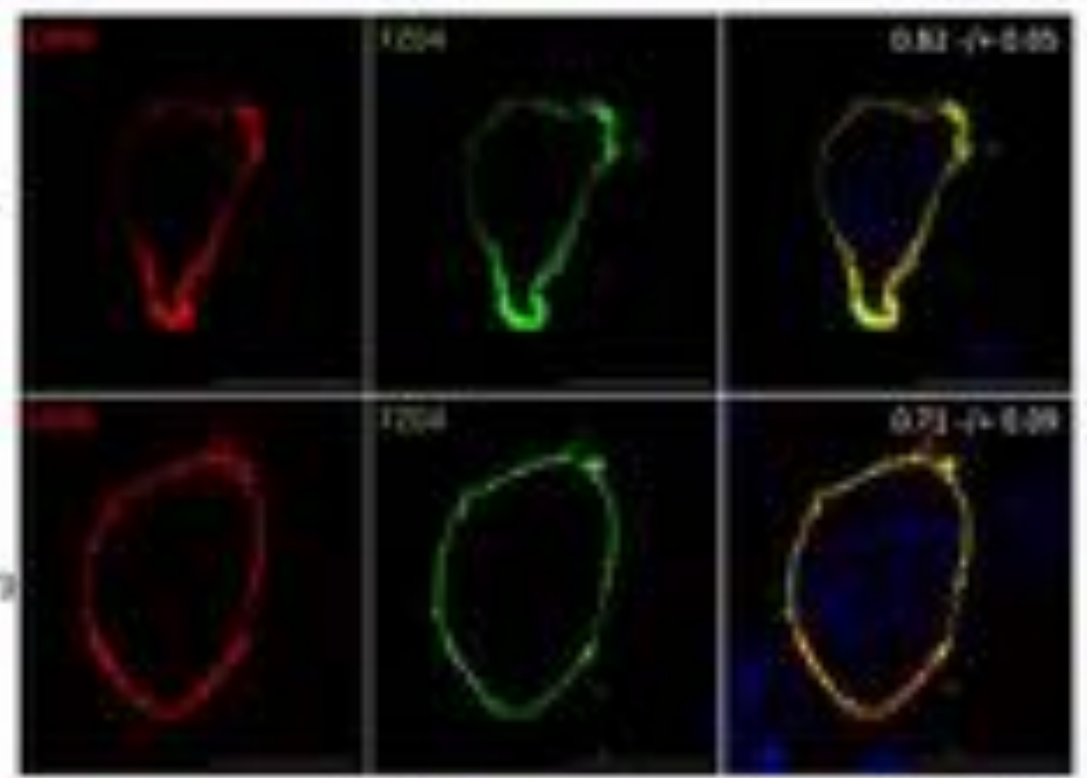

b

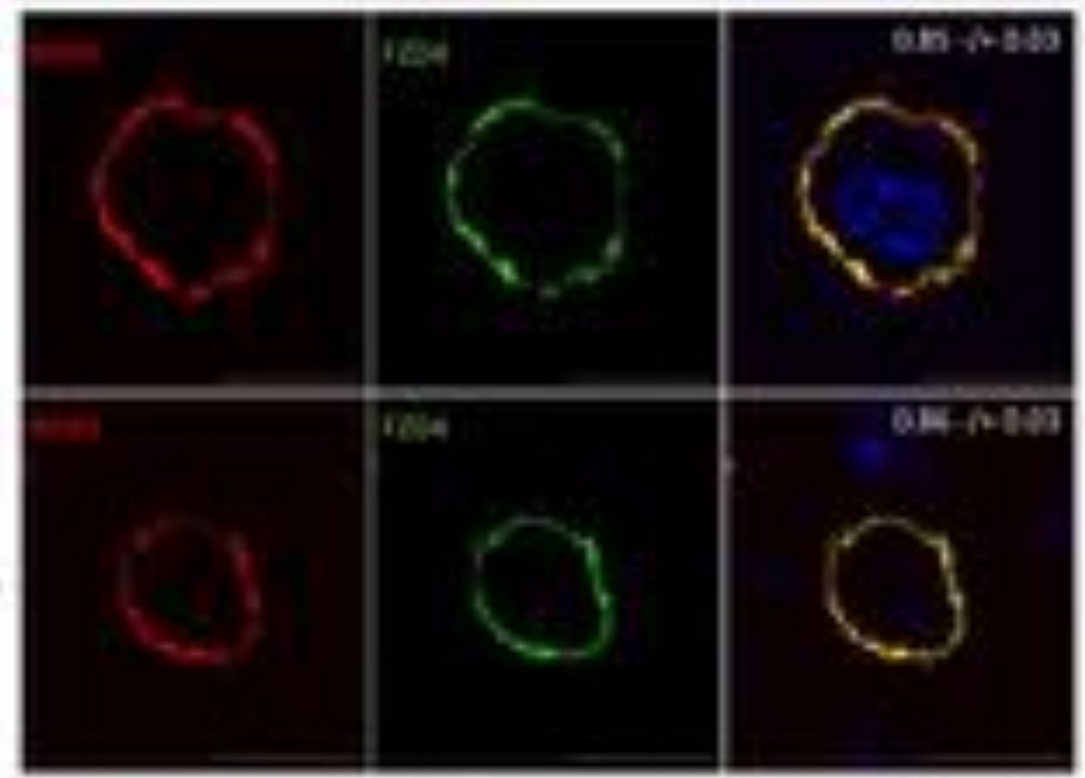

c

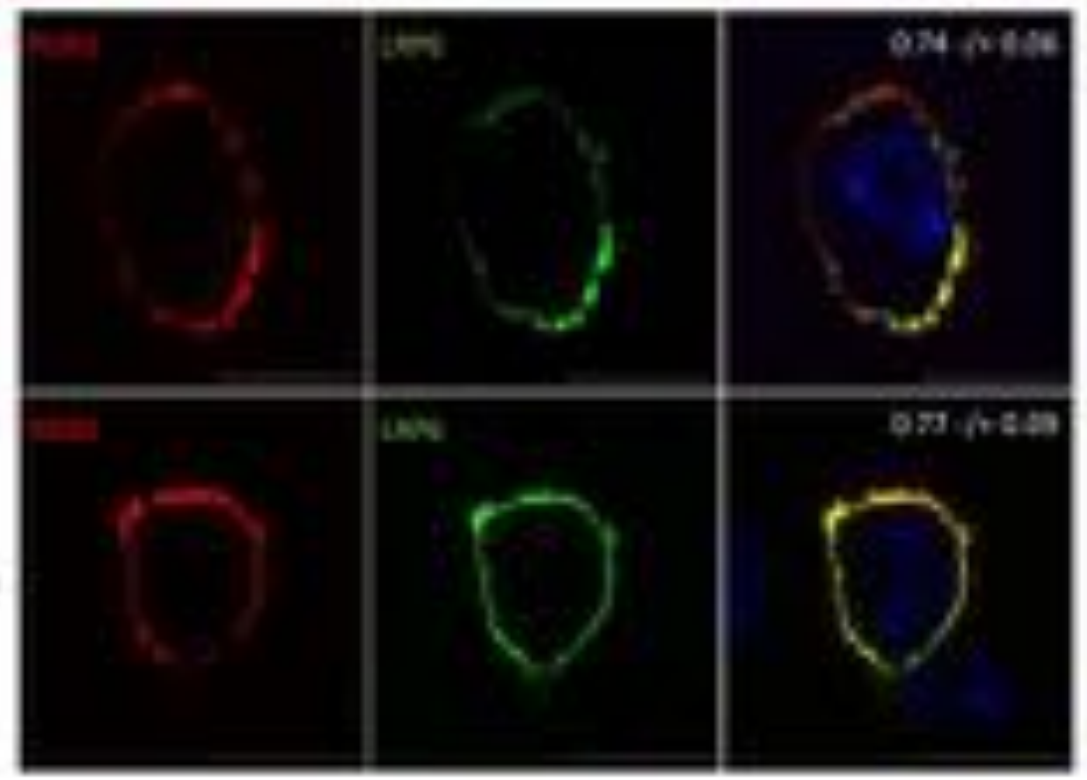

LWha 


\section{lenpouet ai, nie?}

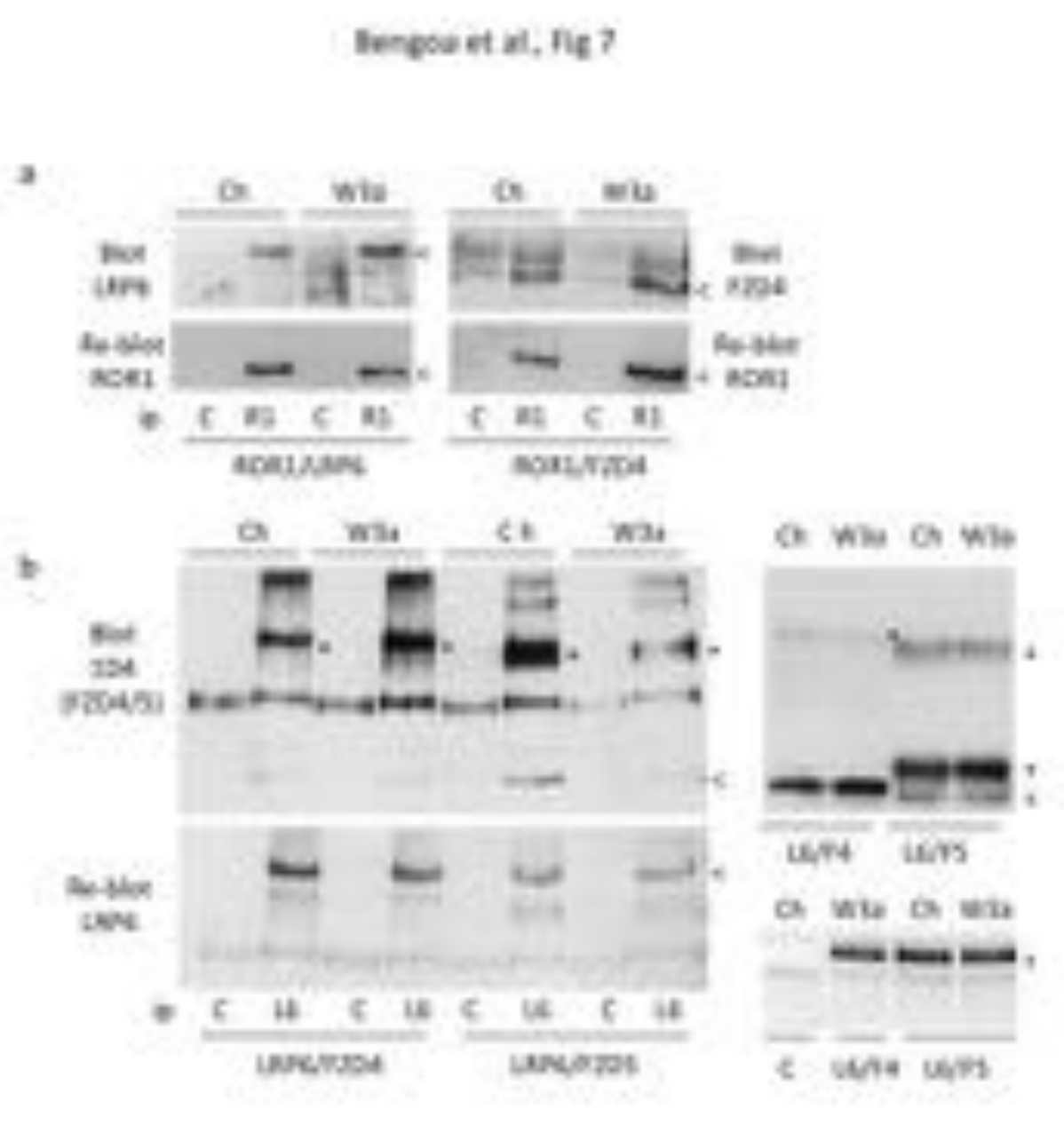

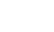
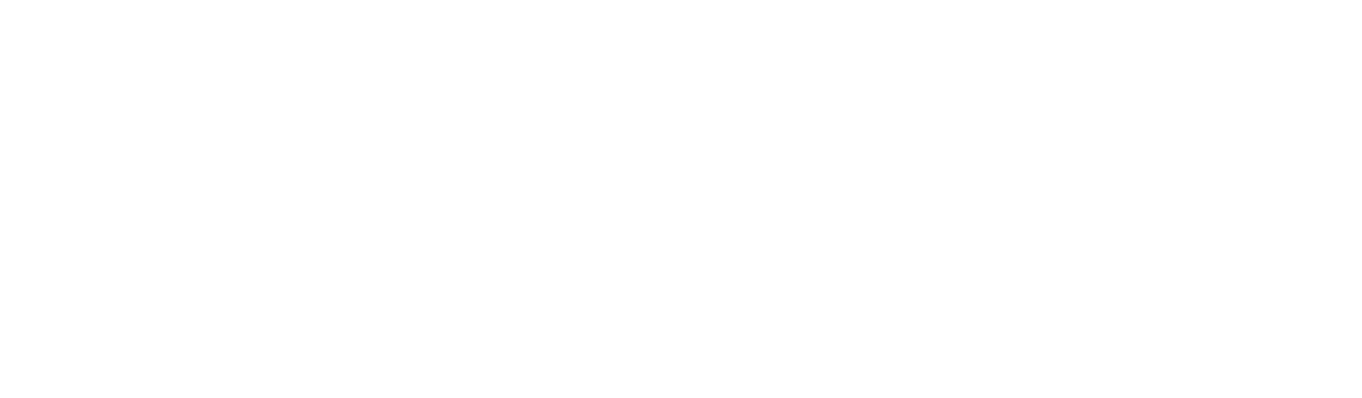

.
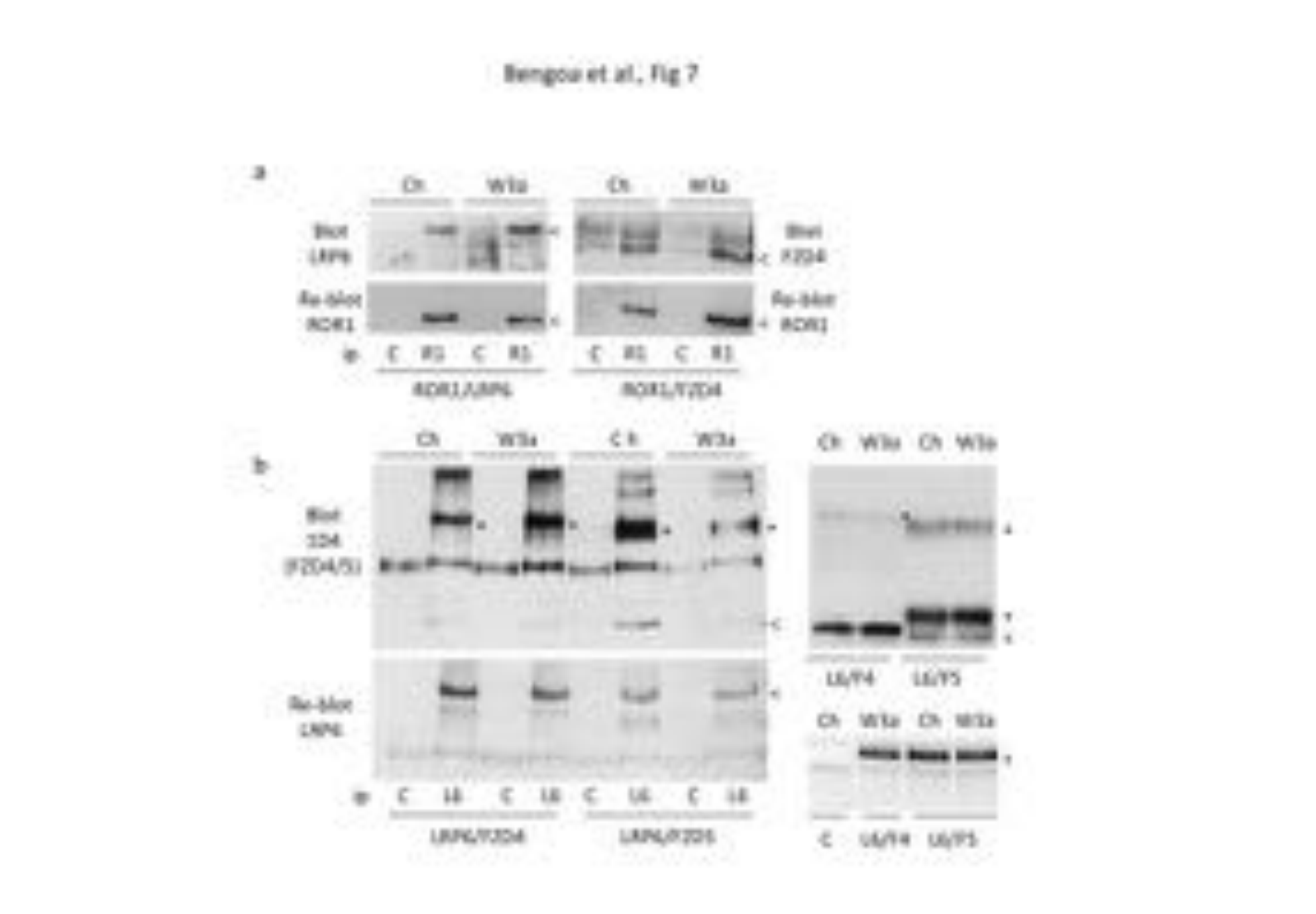

.

.

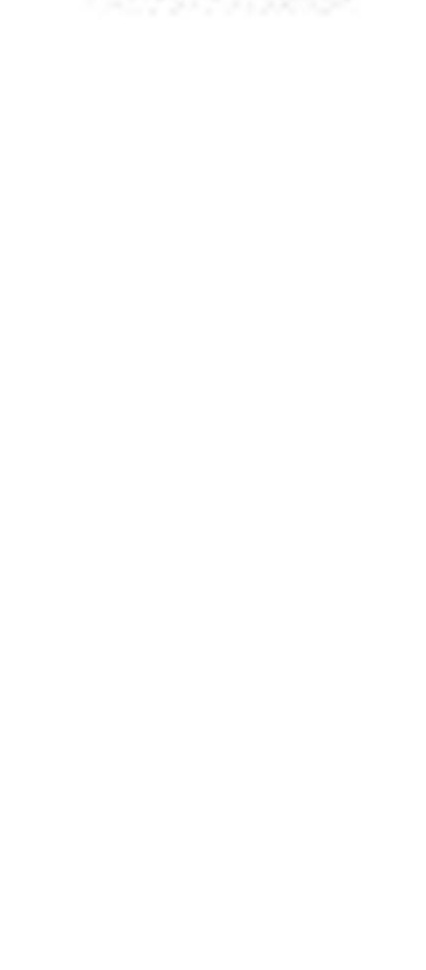


Click here to access/download Supplementary Material

Bengoa 2016 revised Supplementary Tables 1 and 2.docx 
Click here to access/download Supplementary Material

Bengoa 2016 revised Supplementary Tables 3, 4 and 5.docx 